AperTO - Archivio Istituzionale Open Access dell'Università di Torino

\title{
Mean-variance dynamic optimality for DC pension schemes
}

\section{This is the author's manuscript}

Original Citation:

Availability:

This version is available http://hdl.handle.net/2318/1735144

since 2020-08-28T12:47:35Z

Published version:

DOI:10.1007/s13385-020-00226-1

Terms of use:

Open Access

Anyone can freely access the full text of works made available as "Open Access". Works made available under a Creative Commons license can be used according to the terms and conditions of said license. Use of all other works requires consent of the right holder (author or publisher) if not exempted from copyright protection by the applicable law. 


\title{
Mean-variance dynamic optimality for DC pension schemes*
}

\author{
Francesco Menoncin $^{\dagger} \quad$ Elena Vigna ${ }^{\ddagger}$
}

March 11, 2020

\begin{abstract}
In this paper we deal with the mean-variance portfolio selection for a defined contribution (DC) pension fund. Since this problem is time-inconsistent, a number of papers have proposed to tackle it through either a Nash equilibrium approach or a precommitment strategy. Here, we adopt the dynamically optimal approach introduced by Pedersen and Peskir (2017), and we compare the dynamically optimal strategy with the precommitment one. While it is well known that the precommitment strategy is the solution to a target-based problem, we show that the same holds for the dynamically optimal strategy. In particular, the precommitment strategy has a constant target, while the dynamically optimal strategy has a time-varying target whose expectation coincides with the constant target of the previous case. We also show that the expected wealth is the same under the two approaches. Numerical applications show that (i) the median of the risky asset's share is lower for the precommitment than the dynamically optimal strategy; (ii) the amount of money invested in the precommitment risky portfolio is highly more volatile than in the dynamically optimal case; (iii) the variance of wealth is lower with the precommitment strategy than with the dynamically
\end{abstract}

${ }^{*}$ We thank an anonymous referee for valuable comments that allowed us to improve the paper.

$\dagger$ University of Brescia.

${ }^{\ddagger}$ Corresponding author. University of Torino and Collegio Carlo Alberto, Italy. Corso Unione Sovietica 218 bis, 10134, Torino, Italy. E-Mail: elena.vigna@unito.it. Tel. +39011 670 5754. ORCID number: 0000-0002-7188-1875. 
optimal one; (iv) under scenarios of extreme market returns (either good or bad), the dynamically optimal strategy allows a more effective reaction because of the continuous adjustment of the final target.

Keywords. Time inconsistency, dynamic programming, martingale approach, precommitment approach, mean-variance portfolio selection.

JEL classification: C61, D81, G11.

\section{Introduction and motivation}

The risk management of defined contribution (DC) pension schemes is gaining increasing importance in industrialized countries. The population aging is threatening the solvency of Pay As You Go public pension systems, and the largely adopted solution is to enhance the employment-based occupational pension schemes, with an overall preference towards defined contribution schemes rather than defined benefit schemes. The search of the most appropriate portfolio strategy in the accumulation phase is the subject of extensive research in the actuarial and financial literature.

Two common optimization criteria are the maximization of the expected utility of the fund's wealth at retirement, and the mean-variance approach. In this paper, we focus on the latter. An important reason for preferring the mean-variance criterion over the expected utility approach is that it transforms the difficult problem of selecting the individual's riskaversion coefficient of a generic utility function into the easier task of choosing an appropriate final target (see Vigna; 2014). Thus, in the context of DC pension funds the mean-variance approach is more user-friendly and of larger applicability.

It is well known that the mean-variance portfolio selection is a time-inconsistent problem due to the presence of the variance of final wealth in the performance criterion (Zhou and Li; 2000 and Basak and Chabakauri; 2010). The problem of time-inconsistency is commonly approached in three ways: (i) the precommitment approach (Strotz; 1956), (ii) the game-theoretical or Nash equilibrium approach (e.g. Basak and Chabakauri; 2010), and (iii) the dynamically optimal approach introduced by Pedersen and Peskir (2017), which is a 
continuous-time version of the so-called naive approach described by Pollak (1968). ${ }^{1}$

The first two approaches have been widely investigated in the portfolio selection problem for DC pension schemes, while the third one has not been adopted in this context. However, the following short review of pros and cons of these two approaches suggests that none of the existing approaches can be considered "the best one" under all viewpoints, and this conclusion stresses the need to investigate alternative investment strategies. Many of the below mentioned papers go in that direction.

Cui et al. (2017) emphasize that the precommitment approach pursues global objectives, while the Nash equilibrium strategy cares about local interests, and they propose a third selfcoordination policy that aims at balancing global interest and local interests of the decisionmaker. Cong and Oosterlee (2016) show that the precommitment strategy is consistent with an investment target but not with a risk attitude, and vice versa the time-consistent strategy is consistent to a risk attitude but not to an investment target, and they add that "it is hard to say whether being consistent with a target or being consistent with a risk aversion attitude is best." Two papers that attack with a different angle the time inconsistency of the precommitment strategy are Cui et al. (2012) and Shi et al. (2017), who propose a weaker notion of time consistency (neither related to the game theoretical nor to the dynamically optimal approach) and find that, while the precommitment strategy in the continuous time in the presence of a pure diffusive market satisfies it, the same is not true for the multi-period time or in a jump diffusion market; they propose semi-self-financing revised mean-variance strategies that beat the precommitment one.

It is worth noting that some limits of the time-consistent policy of the game theoretical approach have been highlighted too. According to Wang and Forsyth (2011), the timeconsistent policy can be found by applying time consistency constraints on the precommitment strategy, and yields therefore an inferior efficient frontier. Another important drawback of the game theoretical approach is in Bensoussan et al. (2019): they analyze the effect of constraints on the value function of both precommitment and game theoretical approaches, and find the unexpected result that for game theoretical approach the presence of constraints can improve the payoff, while for the precommitment approach this paradox does not occur.

\footnotetext{
${ }^{1}$ In the remaining of the paper, the third approach (and the corresponding investment strategy) will be either called dynamically optimal, or dynamically optimal naive, or simply naive.
} 
Since the risk management problem in a DC pension scheme is a crucial topic in the agenda of welfare systems of many countries, the relevance of investigating strategies alternative to precommitment and Nash equilibrium is evident. In particular, in the existing literature the third approach mentioned above, the naive strategy, has never been adopted in DC pension funds. This paper fills this gap in the literature by adopting the dynamically optimal naive approach to solve a mean-variance portfolio selection problem in a DC pension scheme. By so doing, this paper also provides actuaries with useful insight regarding the application of the naive and the precommitment investment strategies to DC pension funds.

A preview of our results is the following. We find that, similarly to the precommitment approach, also the naive one is equivalent to a target-based approach. Differently from the precommitment approach, the target of the naive strategy is stochastic and moves over time in response to renovated circumstances. In the simple Black and Scholes financial settings with constant parameters we prove that the expectation of the stochastic target associated with the naive strategy coincides with the constant target of the precommitment strategy. In the same settings we also prove that the expected wealth of precommitment and naive strategies coincide. Numerical simulations show that: (i) the median of the risky asset's share is lower for the precommitment than the dynamically optimal strategy; (ii) the amount of money invested in the precommitment risky portfolio is highly more volatile than in the naive case; (iii) the variance of wealth is lower with the precommitment strategy than with the naive one; and (iv) because of the continuous adjustment of the final target, under scenarios of extreme market returns both in the good and in the bad direction, the naive strategy allows a more effective reaction: in a bear market phase it provides a better hedge against losses, while in a bull market phase it allows to exploit the high returns.

The remainder of the paper is structured as follows. In Section 2 we define the financial market. In Section 3 we set the mean-variance problem. In Section 4 we present and compare the precommitment and the dynamically optimal approaches. In Section 5 we derive the optimal portfolios for the two approaches, while in Section 6 we provide further theoretical results about their comparison. Section 7 is devoted to numerical simulations and Section 8 concludes. 


\section{The model}

At time $t_{0} \geq 0$ a worker joins a DC pension scheme whose initial fund is $x_{0} \geq 0$. We assume that from $t_{0}$ till $T>t_{0}$ the worker pays periodic contributions into the fund as a percentage of his salary, and the retirement $T$ is fixed. Therefore, neither the contribution rate nor the retirement date are control variables. ${ }^{2}$ The plan member decides the asset allocation at any time $t \in\left[t_{0}, T\right]$.

We present a general model, in which the uncertainty on an arbitrage free and complete financial market is driven by a set of $s$ stochastic state variables. In the market one riskless asset and $n$ risky assets are listed. As a particular case, we also present a framework where all the state variables are constant and there is only one risky asset following a geometric Brownian motion (so-called Black and Scholes model, Björk; 1998).

\subsection{General model}

The financial market is arbitrage free, complete, frictionless, and continuously open at any time $t \in\left[t_{0}, T\right]$. The risk is described by a set of $n$ independent Brownian motions $W(t)$, defined on the complete filtered probability space $\{\Omega, \mathcal{F}(t), \mathbb{P}\}$, where $\{\mathcal{F}(t)\}_{t \in\left[t_{0}, T\right]}$ is the filtration generated by the Brownian motions and $\mathbb{P}$ is the real-world probability measure. The financial market is described by the following variables:

- $s$ state variables $z(t)$ (with $z\left(t_{0}\right)=z_{0} \in \mathbb{R}^{s}$ known) whose values solve the matrix stochastic differential equation (SDE)

$$
\underset{s \times 1}{d z} \underset{s \times 1}{\mu_{z}(t, z)} \underset{s \times n}{\Omega(t, z)} \underset{n \times 1}{(W}(t)
$$

- one riskless asset whose price $G(t)$ solves the (ordinary) differential equation

$$
d G(t)=G(t) r(t, z) d t
$$

where $r(t, z)$ is the spot instantaneously riskless interest rate;

\footnotetext{
${ }^{2}$ In reality, to some extent the retirement time and in some cases also the contribution rate can be chosen by the worker. However, in this work we focus on the asset allocation only.
} 
- $n$ risky assets whose prices $S(t)$ (with $S\left(t_{0}\right)=s_{0} \in \mathbb{R}^{n}$ known) solve the matrix stochastic differential equation

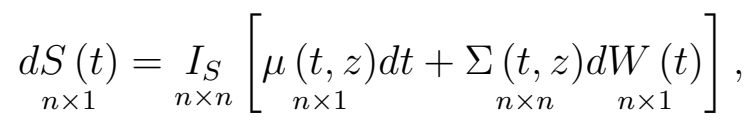

where $I_{S}$ is the $n \times n$ square diagonal matrix gathering the prices $S_{1}, S_{2}, \ldots, S_{n}$.

The drift and diffusion terms in (1) and (2) are assumed to satisfy the usual conditions for the existence and uniqueness of a strong solution to the SDEs.

The absence of arbitrage and completeness imply the existence of a unique risk-neutral equivalent martingale measure $\mathbb{Q}$. This also implies the existence and uniqueness of a market prices of risk $\xi(t, z) \in \mathbb{R}^{n}$ which solves the linear system $\Sigma(t, z) \xi(t, z)=\mu(t, z)-r(t, z) \mathbf{1}$, where 1 is a vector of 1 's (i.e. $\left.\exists \Sigma(t, z)^{-1}\right)$. Assuming that $\xi(t, z)$ satisfies the Novikov's condition, the Girsanov theorem applies and the Wiener processes $d W(t)$ can be rewritten under $\mathbb{Q}$ as follows:

$$
d W^{\mathbb{Q}}(t)=\xi(t, z) d t+d W(t) .
$$

The Radon-Nikodym derivative is (the prime denotes transposition):

$$
m\left(t_{0}, t\right)=e^{-\frac{1}{2} \int_{t_{0}}^{t} \xi(u, z)^{\prime} \xi(u, z) d u-\int_{t_{0}}^{t} \xi(u, z)^{\prime} d W(u)} \Longleftrightarrow\left\{\begin{array}{l}
d m\left(t_{0}, t\right)=-m\left(t_{0}, t\right) \xi(t, z)^{\prime} d W(t), \\
m\left(t_{0}, t_{0}\right)=1 .
\end{array}\right.
$$

Thus, given any $t$-measurable random variable $\Xi(t)$, the following relationship holds true

$$
\mathbb{E}_{t_{0}}^{\mathbb{Q}}[\Xi(t)]=\mathbb{E}_{t_{0}}\left[\Xi(t) \cdot m\left(t_{0}, t\right)\right]
$$

where $\mathbb{E}_{t_{0}}^{\mathbb{Q}}[\bullet]$ and $\mathbb{E}_{t_{0}}[\bullet]$ are the expected values conditioned on $\mathcal{F}\left(t_{0}\right)$ and computed under the risk neutral or the real world probabilities, respectively.

Remark 1. Throughout the paper, the notation $\mathbb{E}_{t_{0}}[\bullet]$ denotes $\mathbb{E}\left[\bullet \mid \mathcal{F}_{t_{0}}\right]$ and is a compact version of the more complete notation $\mathbb{E}_{t_{0}, z_{0}, x_{0}}[\bullet]$.

Let $B(t, T)$ be the price at $t$ of a zero-coupon bond expiring in $T$, and $\sigma_{B}(t, T)$ the (vector) diffusion term of $\frac{d B(t, T)}{B(t, T)}$. It is well known that the so-called "forward probability 
measure" $\left(\mathbb{F}_{T}\right)$ can be defined as follows

$$
d W^{\mathbb{Q}}(t)=\sigma_{B}(t, T) d t+d W^{\mathbb{F}_{T}}(t)
$$

and, given any $T$-measurable random variable $\Xi(T)$, we can write

$$
\mathbb{E}_{t}^{\mathbb{Q}}\left[\Xi(T) e^{-\int_{t}^{T} r(u, z) d u}\right]=\mathbb{E}_{t}^{\mathbb{F}_{T}}[\Xi(T)] \mathbb{E}_{t}^{\mathbb{Q}}\left[e^{-\int_{t}^{T} r(u, z) d u}\right]=\mathbb{E}_{t}^{\mathbb{F}_{T}}[\Xi(T)] B(t, T),
$$

where the new numéraire of the economy is $B(t, T)$ (Björk; 1998). $\mathbb{F}_{T}$ is useful for simplifying the role of contributions in the evolution of the pension fund's wealth.

Remark 2. The forward probability measure is needed to split the expected value of a product into the product of two expected values, as in (6). In this way, also the derivative of the expected value can be written in a much simpler way.

A stochastic contribution $c(t, z)>0$ is continuously paid by the member into the fund's wealth $X(t)$. If $w(t) \in \mathbb{R}^{n}$ contains the monetary amount invested at time $t$ in each risky asset (i.e. a portfolio) and satisfies the usual "admissible" properties (Karatzas and Shreve; 1998), the wealth dynamics are given by the following SDE:

$$
d X(t)=\left(X(t) r(t, z)+c(t, z)+w(t)^{\prime}(\mu(t, z)-r(t, z) \mathbf{1})\right) d t+w(t)^{\prime} \Sigma(t, z) d W(t) .
$$

\subsection{Black and Scholes model, constant salary}

Our general model collapses into the Black and Scholes framework if we assume $\mu_{z}=\mathbf{0}$ and $\Omega=\mathbf{0}$ (where $\mathbf{0}$ is a matrix/vector of zeros), i.e. all the state variables are constant. Accordingly, both the interest rate and the contributions are constant and positive: $r \geq 0$,

$c \geq 0$. Furthermore, in the financial market, we have $n=1$ and both $\mu$ and $\Sigma=\sigma$ are constant. Thus, we can write

$$
\begin{gathered}
d G(t)=G(t) r d t \\
d S(t)=\mu S(t) d t+\sigma S(t) d W(t),
\end{gathered}
$$


and the wealth dynamics is accordingly

$$
d X(t)=(X(t) r+c+w(t)(\mu-r)) d t+w(t) \sigma d W(t) .
$$

\section{The mean-variance problem}

At time $t_{0}$ with initial state variables $z_{0}$ and initial wealth $x_{0}$ the plan member wants to maximize the expected final wealth at retirement, adjusted by the wealth variance that can be interpreted like a risk measure. Specifically, he wants to solve the following mean-variance problem: ${ }^{3}$

$$
\left[\mathcal{P}_{t_{0}, z_{0}, x_{0}}^{M V} \quad \sup _{w} J^{M V}\left(t_{0}, z_{0}, x_{0}, w\right)=\sup _{w}\left\{\mathbb{E}_{t_{0}}[X(T)]-\alpha \mathbb{V}_{t_{0}}[X(T)]\right\},\right.
$$

where the optimization is done over some set of admissible controls, and $\alpha>0$ is a measure of the agent's risk aversion.

It is well known (e.g. Zhou and Li; 2000) that it is not possible to solve the meanvariance problem $\left[\mathcal{P}_{t_{0}, z_{0}, x_{0}}^{M V}\right]$ with dynamic programming, because of the presence of a nonlinear function of expected final wealth in the performance criterion (Björk et al.; 2017). Thus, according to the existing literature, the problem is said to be time-inconsistent.

There are three possible ways to tackle this time-inconsistency: (i) a precommitment approach; (ii) a game theoretical approach; (iii) a dynamically optimal or naive approach. The first gives raise to a time-inconsistent policy, while the last two approaches lead to time-consistent policies.

In the current literature on defined contribution pension schemes, only the first and the second approaches have been thoroughly investigated, see, among others, He and Liang (2013), Yao et al. (2013), Yao et al. (2014), Menoncin and Vigna (2017), Guan and Liang (2015) and $\mathrm{Wu}$ et al. (2015). Instead, the third one has neither been adopted nor analyzed.

In this paper we fill this gap of the literature. In particular, we investigate the adoption of the dynamically optimal naive approach in a defined contribution pension scheme and we

\footnotetext{
${ }^{3}$ We use $\mathbb{V}_{t_{0}}[\bullet]$ as a short notation for $\mathbb{V}_{t_{0}, z_{0}, x_{0}}[\bullet]$, similarly to what we do for the expectation, see Remark 1.
} 
make a comparison with the precommitment approach.

\section{The precommitment and the dynamically optimal naive approaches}

\subsection{Precommitment approach}

Given the initial point $\left(t_{0}, z_{0}, x_{0}\right)$, the so-called precommitment strategy that solves the meanvariance problem $\left[\mathcal{P}_{t_{0}, z_{0}, x_{0}}^{M V}\right]$ in (9) is the control plan $\hat{w}$ that maximizes just $J^{M V}\left(t_{0}, z_{0}, x_{0}, w\right)$. More formally, we can write what follows.

Definition 1. Given the mean-variance problem (9), if there exists a strategy $\hat{w}_{t_{0}, z_{0}, z_{0}}(t, z, x)$, with $(t, z, x) \in\left[t_{0}, T\right] \times \mathbb{R}^{s} \times \mathbb{R}$, that maximizes $J^{M V}\left(t_{0}, z_{0}, x_{0}, w\right)$, i.e., a control map

$$
\hat{w}_{t_{0}, z_{0}, x_{0}}:\left[t_{0}, T\right] \times \mathbb{R}^{s} \times \mathbb{R} \rightarrow \mathbb{R}^{n}
$$

such that

$$
J^{M V}\left(t_{0}, z_{0}, x_{0}, \hat{w}_{t_{0}, z_{0}, x_{0}}\right)=\sup _{w} J^{M V}\left(t_{0}, z_{0}, x_{0}, w\right),
$$

then the strategy $\hat{w}_{t_{0}, z_{0}, x_{0}}(t, z, x)$ for $(t, z, x) \in\left[t_{0}, T\right] \times \mathbb{R}^{s} \times \mathbb{R}$ is called precommitment strategy.

The precommitment strategy for $\left[\mathcal{P}_{t_{0}, z_{0}, x_{0}}^{M V}\right]$ in both models of Sections 2.1 and 2.2 exists and is known in closed form (Vigna; 2014, and Menoncin and Vigna; 2017).

\subsection{Dynamically optimal or naive approach}

The dynamically optimal approach introduced by Pedersen and Peskir (2017) is the continuoustime version of the naive approach described by Pollak (1968). This approach can be easily defined from the precommitment approach. We illustrate the construction of the dynamically optimal strategy for the mean-variance problem $\left[\mathcal{P}_{t_{0}, z_{0}, x_{0}}^{M V}\right]$ in three steps. 
Step 1. Assume that for the initial point $\left(t_{0}, z_{0}, x_{0}\right)$ there exists the precommitment strategy

$$
\hat{w}_{t_{0}, z_{0}, x_{0}}:\left[t_{0}, T\right] \times \mathbb{R}^{s} \times \mathbb{R} \rightarrow \mathbb{R}^{n}
$$

that maximizes the criterion $J^{M V}\left(t_{0}, z_{0}, x_{0}, w\right)$.

Step 2. Define the new control map

$$
\tilde{w}:\left[t_{0}, T\right] \times \mathbb{R}^{s} \times \mathbb{R} \rightarrow \mathbb{R}^{n}
$$

as follows

$$
\tilde{w}(t, z, x):=\hat{w}_{t, z, x}(t, z, x) \quad \forall(t, z, x) \in\left[t_{0}, T\right] \times \mathbb{R}^{s} \times \mathbb{R},
$$

where the right hand side of $(13)$ is obtained by replacing $\left(t_{0}, z_{0}, x_{0}\right)$ with $(t, z, x)$ in the function (11).

Step 3. The strategy $\tilde{w}(t, z, x)$ for $(t, z, x) \in\left[t_{0}, T\right] \times \mathbb{R}^{s} \times \mathbb{R}$ given by (13) is called the dynamically optimal or naive strategy.

To put it in simple terms, the dynamically optimal strategy is obtained by replacing $\left(t_{0}, z_{0}, x_{0}\right)$ with $(t, z, x)$ in the precommitment strategy. Therefore, the calculation of the dynamically optimal naive strategy for the models of Sections 2.1 and 2.2 is straightforward.

\subsection{Link between the two approaches}

There is a strict link between the dynamically optimal naive and the precommitment approaches: $^{4}$

- At time $t_{0}$ with wealth $x_{0}$ the dynamically optimal naive investor and the precommitted investor play the same strategy $\hat{w}_{t_{0}, z_{0}, x_{0}}\left(t_{0}, z_{0}, x_{0}\right)$, and they face the same problem $\left[\mathcal{P}_{t_{0}, z_{0}, x_{0}}^{M V}\right]$.

\footnotetext{
${ }^{4}$ To improve the readability and the interpretation, in what follows we will sometimes refer only to wealth (that is the only controlled state variable), and will ignore the remaining state variables. In the special case of the Black and Scholes market, where there are no state variables other than wealth, this turns out to be correct.
} 
- When time passes by, at time $\left.t \in] t_{0}, T\right]$ with wealth $x$ the naive investor faces problem $\left[\mathcal{P}_{t, z, x}^{M V}\right]$ and solves it with the precommitment approach at time $t$, as if his initial point were $(t, z, x)$.

- In fact, the naive investor plays $\hat{w}_{t, z, x}(t, z, x)$, that would be the initial control played by an investor who, starting at time $t$ with wealth $x$, wants to solve Problem $\left[P_{t, z, x}^{M V}\right]$ over the time horizon $[t, T]$ with the precommitment approach.

- Then, the intuition is that the dynamically optimal investor can be seen as the continuous reincarnation of the precommitted investor.

- Such an investor is called eitherdynamically optimal by Pedersen and Peskir (2017) or naive by Pollak (1968).

\subsection{A target-based approach}

Both strategies that we have presented in the previous sections, can be interpreted as two particular cases of target-based problems. Indeed, it is possible to prove (Zhou and Li; 2000, and Vigna; 2014) that a target $\gamma_{t_{0}, z_{0}, x_{0}} \in \mathbb{R}$ exists such that at any time $t \in\left[t_{0}, T\right]$ with wealth $x$ the precommitted investor plays the strategy that minimizes the following criterion

$$
\mathbb{E}_{t}\left[\left(X(T)-\gamma_{t_{0}, z_{0}, x_{0}}\right)^{2}\right] .
$$

In other words, the precommitted investor plays the strategy that makes the final wealth as close as possible to the target $\gamma_{t_{0}, z_{0}, x_{0}}$. In this case the target is constant over time. Instead, it can be proved that the naive investor at time $t$ with wealth $x$ plays the strategy that minimizes the following criterion

$$
\mathbb{E}_{t}\left[\left(X(T)-\gamma_{t, z, x}\right)^{2}\right]
$$

In other words, the dynamically optimal naive investor plays the strategy that makes the final wealth as close as possible to the target $\gamma_{t, z, x}$. Nevertheless, in this case the target is time-varying and it also depends on the wealth $x$ achieved at time $t$ (i.e. $\gamma_{t, z, x} \neq \gamma_{t_{0}, z_{0}, x_{0}}$ ). In the next section we show the value of the target for the two approaches. 


\section{The precommitment and the naive portfolios}

Since the naive portfolio is obtained from the precommitment one, we first present the precommitment strategy.

\subsection{The precommitment portfolio}

After Zhou and Li (2000), it is known that the mean-variance problem (9) can be recast as a target problem in the following form

$$
\inf _{w} J\left(t_{0}, z_{0}, x_{0}, w\right)=\inf _{w} \mathbb{E}_{t_{0}}\left[\frac{1}{2}\left(X(T)-\gamma_{t_{0}, z_{0}, x_{0}}\right)^{2}\right],
$$

in which

$$
\begin{gathered}
\gamma_{t_{0}, z_{0}, x_{0}}=\frac{x_{0}+\int_{t_{0}}^{T} \mathbb{E}_{t_{0}}^{\mathbb{F}_{s}}[c(s, z)] B\left(t_{0}, s\right) d s}{B\left(t_{0}, T\right)}+\frac{1}{2 \alpha} \frac{\mathbb{E}_{t_{0}}\left[e^{2 \Phi\left(t_{0}, T\right)}\right]}{B\left(t_{0}, T\right)^{2}}, \\
\Phi\left(t_{0}, T\right)=-\int_{t_{0}}^{T} r(u, z) d u-\frac{1}{2} \int_{t_{0}}^{T} \xi(u, z)^{\prime} \xi(u, z) d u-\int_{t_{0}}^{T} \xi(u, z)^{\prime} d W(u) .
\end{gathered}
$$

The form of the target is worth a comment. The first term of (15) coincides with the forward price of a floating versus fix swap. Let us assume that the fund wants to exchange its future stochastic cash flows till time $T$ with a fixed amount $x_{0}^{T}$ of money to be paid in $T$. This forward price $x_{0}^{T}$ is a kind of certain equivalent for the whole cash flows of the fund, and, with a light abuse of terminology, we will call it certain equivalent in $T$, or $T$-certain equivalent. The value of $x_{0}^{T}$ must satisfy the following pricing equation:

$$
0=\mathbb{E}_{t_{0}}^{\mathbb{Q}}\left[x_{0}^{T} e^{-\int_{t_{0}}^{T} r(u, z) d u}-\left(x_{0}+\int_{t_{0}}^{T} c(s, z) e^{-\int_{t_{0}}^{s} r(u, z) d u} d s\right)\right]
$$

Through this contract, the fund pays its initial wealth $x_{0}$ and all the subsequent payments $c(t, z)$ to its counterpart, and, in exchange, it receives, at maturity $T$, a constant amount of money $x_{0}^{T}$ that solves (17). If we simplify this equation, the final result is

$$
x_{0}^{T}=\frac{x_{0}+\int_{t_{0}}^{T} \mathbb{E}_{t_{0}}^{\mathbb{F}_{s}}[c(s, z)] B\left(t_{0}, s\right) d s}{B\left(t_{0}, T\right)} .
$$


The target $\gamma_{t_{0}, z_{0}, x_{0}}$ is greater than the $T$-certain equivalent, in fact:

$$
\gamma_{t_{0}, z_{0}, x_{0}}=x_{0}^{T}+\frac{1}{2 \alpha} \frac{\mathbb{E}_{t_{0}}\left[e^{2 \Phi\left(t_{0}, T\right)}\right]}{B\left(t_{0}, T\right)^{2}}
$$

and the amount that is added to $x_{0}^{T}$ for obtaining the target is a function of the risk aversion $\alpha$. If the risk aversion is very high, then the fund will try to stay as close as possible to the $T$-certain equivalent, while if $\alpha$ is sufficiently low, the target will depart substantially from the $T$-certain equivalent.

In the Black and Scholes case, with $c$ and $r$ constant, the $T$-certain equivalent becomes

$$
x_{0}^{T}=\frac{x_{0}+c \int_{t_{0}}^{T} e^{-r\left(s-t_{0}\right)} d s}{e^{-r\left(T-t_{0}\right)}}=x_{0} e^{r\left(T-t_{0}\right)}+c \frac{e^{r\left(T-t_{0}\right)}-1}{r},
$$

which coincides with the compounded value at the riskless rate $r$ of initial wealth and contributions (i.e., the amount of money that could be obtained at time $T$ by investing initial wealth $x_{0}$ and future contributions in the riskless asset). In the same framework, the target is

$$
\gamma_{t_{0}, x_{0}}=x_{0} e^{r\left(T-t_{0}\right)}+c \frac{e^{r\left(T-t_{0}\right)}-1}{r}+\frac{1}{2 \alpha} e^{\xi^{2}\left(T-t_{0}\right)},
$$

where $\xi=\frac{\mu-r}{\sigma}$ is the Sharpe ratio of the risky asset.

The solution to problem (14) is provided in the following proposition.

Proposition 1. The optimal strategy of Problem (14), that coincides with the precommitment solution to Problem (9), is

$$
\begin{aligned}
\hat{w}_{t_{0}, z_{0}, x_{0}}(t, z, x) & =B(t, T)\left(\gamma_{t_{0}, z_{0}, x_{0}}-x_{t}^{T}\right)\left(\Sigma^{\prime}\right)^{-1} \xi \\
& +\left(\Sigma^{\prime}\right)^{-1} \Omega^{\prime} \frac{\partial\left(B(t, T)\left(\gamma_{t_{0}, z_{0}, x_{0}}-x_{t}^{T}\right)\right)}{\partial z(t)} \\
& -B(t, T)\left(\gamma_{t_{0}, z_{0}, x_{0}}-x_{t}^{T}\right)\left(\Sigma^{\prime}\right)^{-1} \Omega^{\prime} \frac{\partial \ln \mathbb{E}_{t}\left[e^{2 \Phi(t, T)}\right]}{\partial z(t)}
\end{aligned}
$$


where $x_{t}^{T}$ is the $T$-certain equivalent at time $t$ :

$$
x_{t}^{T}=\frac{x+\int_{t}^{T} \mathbb{E}_{t}^{\mathbb{F} s}[c(s, z)] B(t, s) d s}{B(t, T)}
$$

and $\gamma_{t_{0}, z_{0}, x_{0}}$ and $\Phi\left(t_{0}, T\right)$ are given in (15) and (16), respectively.

Proof. This result has been proven previously by Menoncin and Vigna (2017). For the reader's convenience, we provide the proof here in the Appendix (A).

The optimal portfolio is formed by three components.

1. A speculative component proportional to the ratio between the market price of risk $\xi$ and the diffusion matrix $\Sigma$. This component also contains the distance between the initial target $\gamma_{t_{0}, z_{0}, x_{0}}$ and the $T$-certain equivalent (21) at time $t$.

2. A hedging component that hedges the fund against the stochastic changes in the $T$ certain equivalent $x_{t}^{T}$. Actually, $x_{t}^{T}$ is a stochastic variable since it is a conditional expected value. This portfolio component is needed because the state variables are stochastic (i.e. $\Omega \neq \mathbf{0}$ ) and is proportional to the correlation between the asset prices and the state variables. In fact, the matrix $\left(\Sigma^{\prime}\right)^{-1} \Omega^{\prime}$ can be written as

$$
\left(\Sigma^{\prime}\right)^{-1} \Omega^{\prime}=\left(\Sigma^{\prime}\right)^{-1} \Sigma^{-1} \Sigma \Omega^{\prime}=\left(\Sigma \Sigma^{\prime}\right)^{-1} \Sigma \Omega^{\prime}
$$

where $\left(\Sigma \Sigma^{\prime}\right)^{-1}$ is the inverse of the variance covariance matrix, while $\Sigma \Omega^{\prime}$ is the matrix that contains the covariances between the asset prices and the state variables. Thus, the term $\left(\Sigma^{\prime}\right)^{-1} \Omega^{\prime}$ can be interpreted as a kind of beta ratio between the market and the state variables.

3. The last hedging component is again proportional to the distance between the initial target $\gamma_{t_{0}, z_{0}, x_{0}}$ and the $T$-certain equivalent $x_{t}^{T}$. Nevertheless, this time, the portfolio component hedges against the stochastic changes in the discount factor $\mathbb{E}_{t}\left[e^{2 \Phi(t, T)}\right]$. In particular, the portfolio contains the semi-elasticity of this discount factor with respect to the state variables. 


\section{Black and Scholes model}

The precommitment strategy for the Black and Scholes model of Section 2.2 is ${ }^{5}$

$$
\hat{w}_{t_{0}, x_{0}}(t, x)=\frac{\xi}{\sigma}\left[\gamma_{t_{0}, x_{0}} e^{-r(T-t)}-x-c \frac{1-e^{-r(T-t)}}{r}\right]
$$

in which $\gamma_{t_{0}, x_{0}}$ is given by (19).

Note that in the Black and Scholes case, the hedging portfolio components do not play any role, since all the state variables $(r$ and $c$ ) are constant (i.e. $\Omega=\mathbf{0}$ ).

\subsection{Dynamically optimal naive approach}

The dynamically optimal naive strategy for the general model of Section 2.1 is obtained by

substituting $\gamma_{t_{0}, z_{0}, x_{0}}$ with $\gamma_{t, z, x}$. In particular, given the value of $\gamma_{t_{0}, z_{0}, x_{0}}$ in (15), we can write

$$
\gamma_{t, z, x}=\frac{1}{2 \alpha} \frac{\mathbb{E}_{t}\left[e^{2 \Phi(t, T)}\right]}{B(t, T)^{2}}+\frac{x+\int_{t}^{T} \mathbb{E}_{t}^{\mathbb{F} s}[c(s, z)] B(t, s) d s}{B(t, T)}
$$

or

$$
\gamma_{t, z, x}=x_{t}^{T}+\frac{1}{2 \alpha} \frac{\mathbb{E}_{t}\left[e^{2 \Phi(t, T)}\right]}{B(t, T)^{2}},
$$

and, accordingly, the dynamically optimal naive strategy is

$$
\begin{aligned}
\tilde{w}(t, z, x) & =\frac{1}{2 \alpha} \frac{\mathbb{E}_{t}\left[e^{2 \Phi(t, T)}\right]}{B(t, T)}\left(\Sigma^{\prime}\right)^{-1} \xi \\
& +\left(\Sigma^{\prime}\right)^{-1} \Omega^{\prime}\left(\gamma_{t, x} \frac{\partial B(t, T)}{\partial z(t)}-\frac{\partial \int_{t}^{T} \mathbb{E}_{t}^{\mathbb{F}_{s}}[c(s, z)] B(t, s) d s}{\partial z(t)}\right) \\
& -\frac{1}{2 \alpha} \frac{\mathbb{E}_{t}\left[e^{2 \Phi(t, T)}\right]}{B(t, T)}\left(\Sigma^{\prime}\right)^{-1} \Omega^{\prime} \frac{\partial \ln \mathbb{E}_{t}\left[e^{2 \Phi(t, T)}\right]}{\partial z(t)} .
\end{aligned}
$$

We note what follows.

1. The speculative portfolio component does not depend any longer on the contributions.

\footnotetext{
${ }^{5}$ In the Black and Scholes model $s=0$ and there is no $z$ variable.
} 
In fact, the state variable $c(t, z)$ does not appear in the portfolio component containing $\left(\Sigma^{\prime}\right)^{-1} \xi$.

2. The second portfolio component that hedges against the stochastic changes in the $T$-certain equivalent $x_{t}^{T}$, instead, still depends on contributions.

3. The last hedging portfolio component does not contain contributions.

\section{Black and Scholes model}

The dynamically optimal naive strategy for the Black and Scholes model of Section 2.2 is obtained by substituting $(t, x)$ to $\left(t_{0}, x_{0}\right)$ in $(22)$, and is:

$$
\tilde{w}(t, x)=\frac{\xi}{\sigma} \frac{1}{2 \alpha} e^{\left(\xi^{2}-r\right)(T-t)}, \quad \forall(t, x) \in\left[t_{0}, T\right] \times \mathbb{R},
$$

in which we see that the amount invested in the risky asset at time $t$ does depend on $t$ but does not depend on the fund level $x$ at time $t$.

Under the hypothesis that the variables $\xi$ and $\sigma$ are positive, we can see that the amount of money optimally invested in the risky asset $\tilde{w}(t, x)$, is either increasing or decreasing over time depending on the sign of the difference $\xi^{2}-r$. The sign of this difference is not obvious (recall that $\xi=\frac{\mu-r}{\sigma}$ ). When the interest rate is "sufficiently" high (low) the difference $\xi^{2}-r$ is negative (positive), and the amount of money invested in the risky asset increases (decreases) over time.

\section{Black and Scholes case: two theoretical results}

In this section we present two theoretical results holding in the Black and Scholes case that shed further light on the interactions between the precommitment and the dynamically optimal approaches.

The first result is that, although the precommitment and the naive strategies are substantially different, the expected value of the corresponding wealth is the same at any time. 
Proposition 2. In the Black and Scholes financial market, let $\hat{X}(t)$ and $\tilde{X}(t)$ denote the wealth at time $t$ under adoption of the precommitment and dynamically optimal naive strategy, respectively. Then,

$$
\mathbb{E}_{t_{0}}[\hat{X}(t)]=\mathbb{E}_{t_{0}}[\tilde{X}(t)] \quad \forall t \in\left[t_{0}, T\right] .
$$

Proof. See Appendix (B).

The second result refers to the connection between the constant target of the precommitment approach and the moving target of the dynamically optimal naive approach. In the precommitment approach the fund's wealth at time $T$ is optimally set as close as possible to a constant target decided at time $t_{0}$ and given the initial wealth $x_{0}$. Instead, in the naive strategy the fund's wealth at time $T$ is optimally set as close as possible to a time-varying target that depends on both the current time $t$ and the current wealth $x$. Interestingly, we find that the expectation at time $t_{0}$ of the stochastic time-varying target relative to the dynamically optimal strategy coincides with the constant target.

Proposition 3. In the Black and Scholes financial market, if $\tilde{X}(t)$ denotes the wealth at time $t$ under adoption of the dynamically optimal naive strategy with $\tilde{X}\left(t_{0}\right)=x_{0}$, then

$$
\mathbb{E}_{t_{0}}\left[\gamma_{t, \tilde{X}(t)}\right]=\gamma_{t_{0}, x_{0}} \quad \forall t \in\left[t_{0}, T\right] .
$$

In other words, the target process $\gamma_{t, \tilde{X}(t)}$ is a martingale.

Proof. See Appendix (C).

Then, the similarities between the two strategies are twofold. Standing at time $t_{0}$, over time the two strategies produce the same expected wealth. Moreover, standing at time $t_{0}$, the final target pursued at every time $t$ remains on average the same.

It is then important to simulate and compare the actual behavior over time of the two strategies and the corresponding wealths, in order to identify those differences that cannot be captured on average. 


\section{Simulations}

In the Black and Scholes model we have run 1000 Monte Carlo simulations with weekly discretisation for both the precommitment and the naive strategy and made a comparison between the two strategies with respect to the behavior over time of several quantities, such as the optimal portfolio and the optimal wealth. We have also investigated the distribution of the time-varying targets relative to the naive approach and compared them with the constant target of the precommitment approach.

The parameters for the simulations are

- $t_{0}=0$, and $T=20$ : we assume that the financial horizon is 20 years;

- $x_{0}=1$ : the initial wealth can of course be scaled for taking into account any other wealth level;

- $c=0.1$ : we assume that the contribution is a percentage of the initial wealth (in this example 10\%);

- $r=3 \%, \mu=8 \%$, and $\xi=\frac{1}{3}$, which imply a volatility $\sigma=0.15$.

With these data, the $T$-certain equivalent $x_{0}^{T}$ is

$$
x_{0}^{T}=x_{0} e^{r\left(T-t_{0}\right)}+c \frac{e^{r\left(T-t_{0}\right)}-1}{r}=4.562515,
$$

while

$$
\frac{1}{2 \alpha} e^{\xi^{2}\left(T-t_{0}\right)}=\frac{4.613907}{\alpha} .
$$

Thus, the initial target is

$$
\gamma_{t_{0}, x_{0}}=x_{0}^{T}+\frac{1}{2 \alpha} e^{\xi^{2}\left(T-t_{0}\right)}=4.562515+\frac{4.613907}{\alpha} .
$$

If we want the target to be 1.2 times the $T$-certain equivalent $x_{0}^{T}$, we have

$$
4.562515+\frac{4.613907}{\alpha}=1.2 \times 4.562515
$$


from which $\alpha=5.0563$, and so

$$
\gamma_{t_{0}, x_{0}}=\gamma_{0,1}=5.475 .
$$

In what follows, we report the results of the simulations for the wealth, the optimal portfolio, and the time-varying target.

Figure 1 reports the statistics for the precommitment $(\mathrm{PC})$ and the naive $(\mathrm{N})$ wealth. The graph on top reports the mean and mean plus/minus twice the standard deviation for both approaches. The bottom left graph reports the minimum, the maximum, and the $5^{\text {th }}$, $25^{\text {th }}, 50^{\text {th }}, 75^{\text {th }}$ and $95^{\text {th }}$ percentiles of PC-wealth, while the graph on the right reports the same measures for the N-wealth.

Figure 2 reports the behavior of the precommitment and naive wealth in the four extreme cases: the best and the worst cases for the final PC-wealth and the best and the worst cases for the final $\mathrm{N}$-wealth. It also reports the path of the price of the risky asset in those extreme cases, as well as the time-varying target of the naive approach.

Figure 3 reports the statistics for the precommitment and the naive optimal investments in the risky asset (i.e. the optimal strategies). The graph on top reports the median for both strategies. The bottom left graph reports the minimum, the maximum, and the $5^{\text {th }}$, $25^{\text {th }}, 50^{\text {th }}, 75^{\text {th }}$ and $95^{\text {th }}$ percentiles of PC-strategy, while the graph on the right reports the same measures for the N-strategy.

Finally, Figure 4 reports the statistics of the time-varying targets for the naive approach, $\gamma_{t, x}$, as well as the constant target for the precommitment approach, $\gamma_{t_{0}, x_{0}}$. 

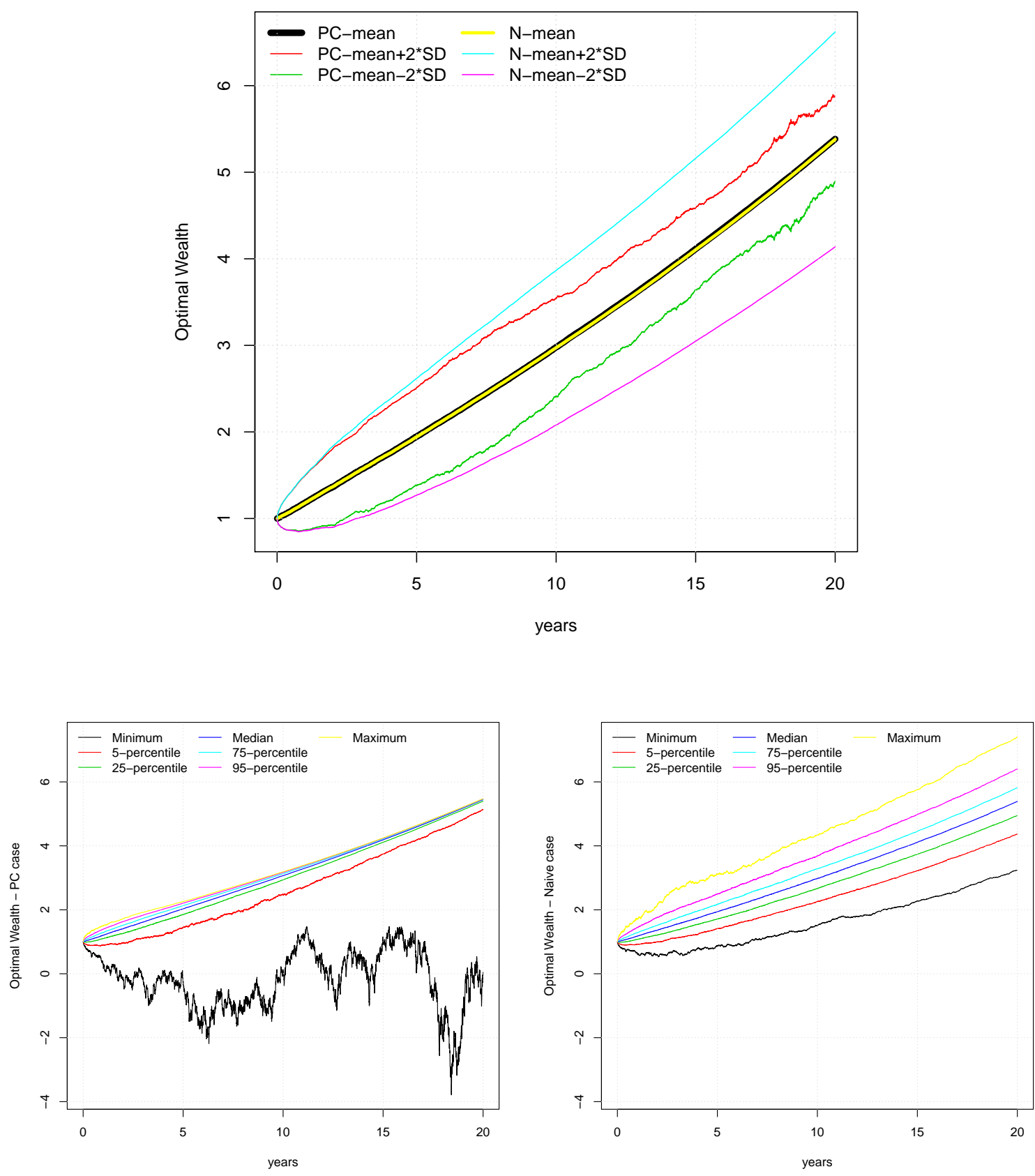

Figure 1: Precommitment (PC) and naive (N) wealths. Top graph: mean and mean \pm 2 standard deviation. Bottom-left graph: statistics of $\mathrm{PC}$-wealth. Bottom-right graph: statistics of N-wealth. 

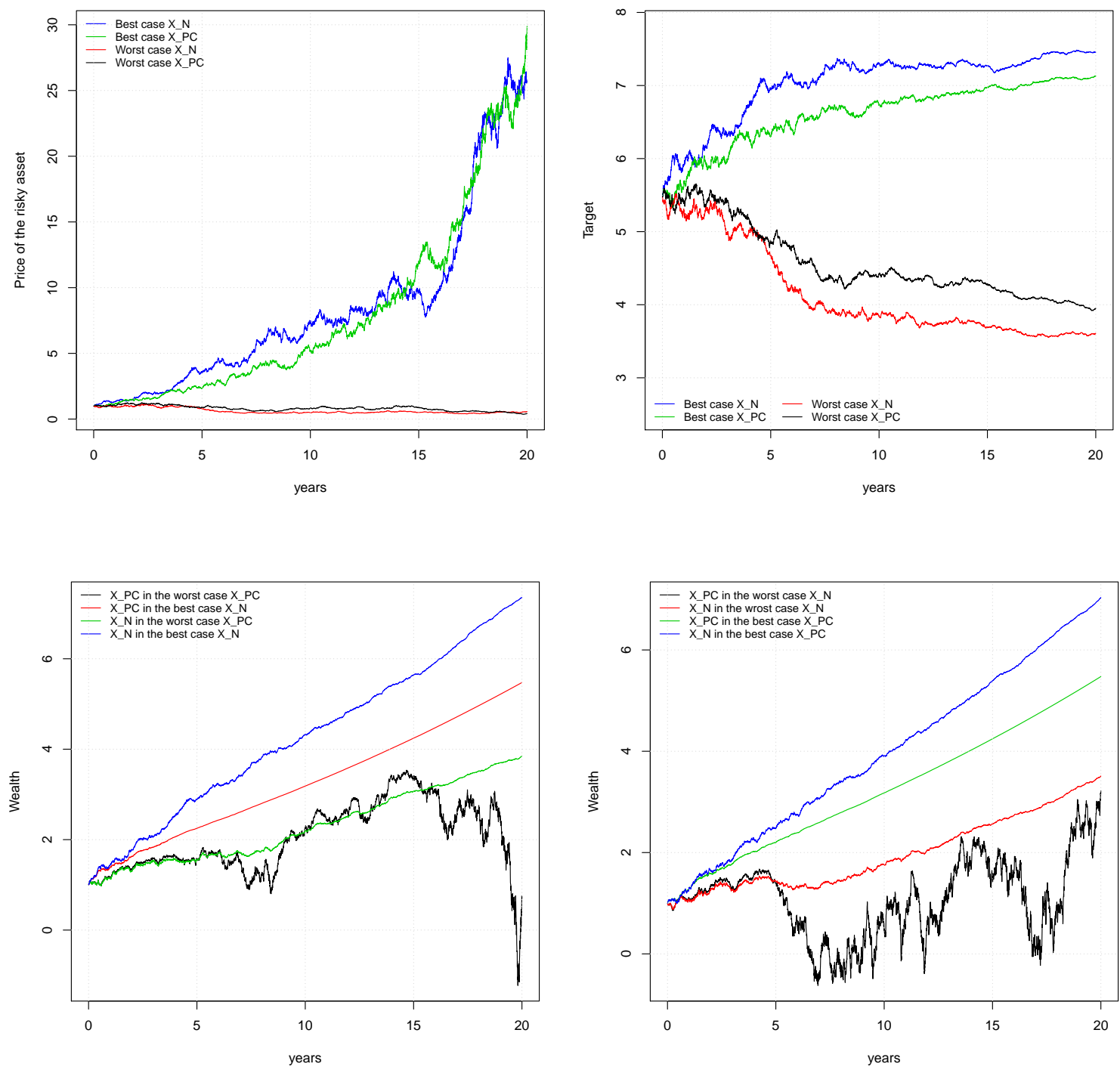

Figure 2: Extreme scenarios and corresponding wealth evolution. Top-left graph: price of risky asset in the four extreme scenarios. Top-right graph: time-varying target for naive approach in the four extreme scenarios. Bottom-left graph: PC- and N- wealths in the best scenario for $\mathrm{N}$-wealth and the worst scenario for PC-wealth. Bottom-right graph: PC- and $\mathrm{N}$ - wealths in the best scenario for PC-wealth and the worst scenario for N-wealth. 

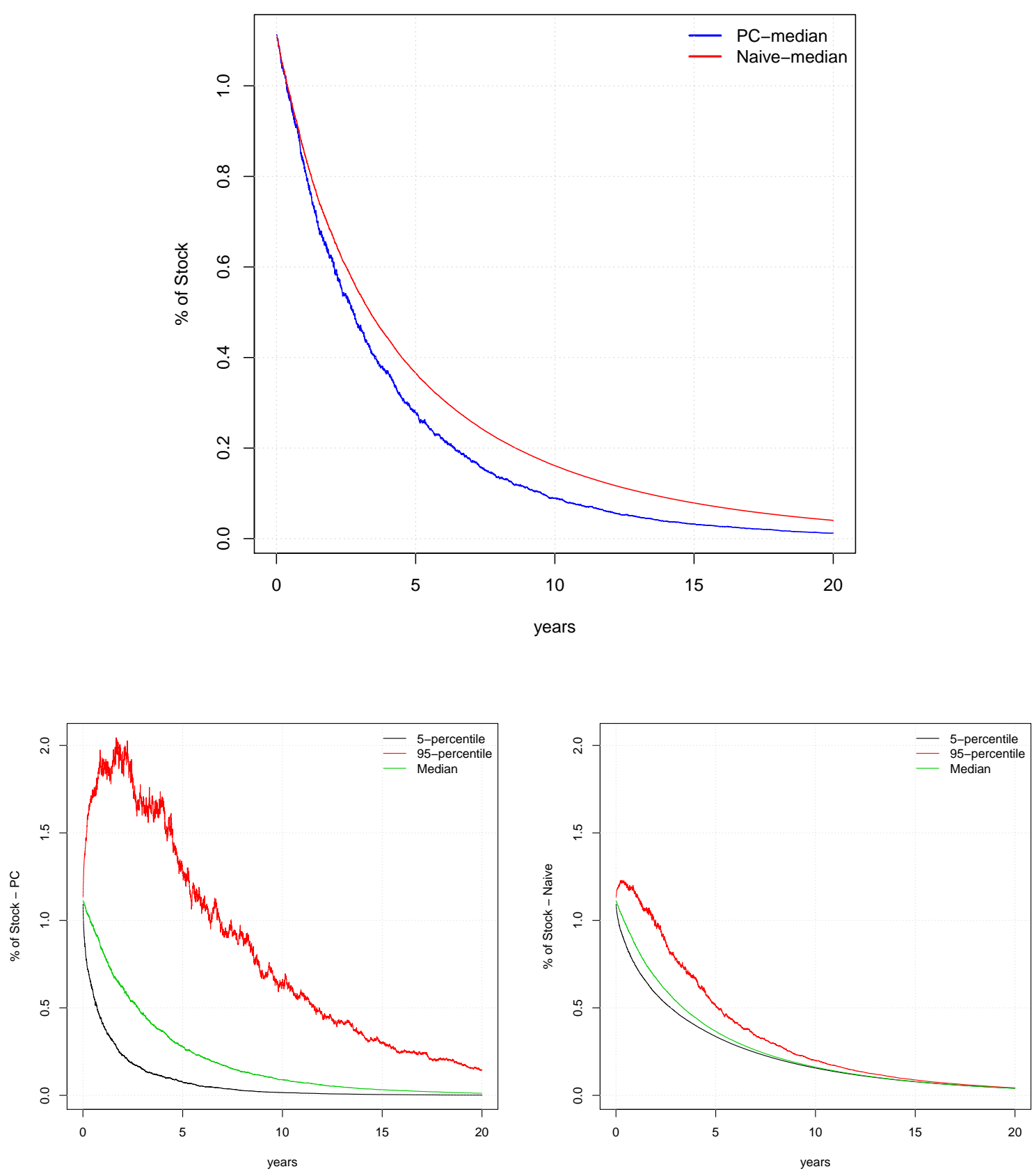

Figure 3: Optimal stock share of precommitment $(\mathrm{PC})$ and naive $(\mathrm{N})$ strategies. Top graph: median of both PC and N. Bottom-left graph: statistics of PC-strategy. Bottom-right graph: statistics of N-strategy. 


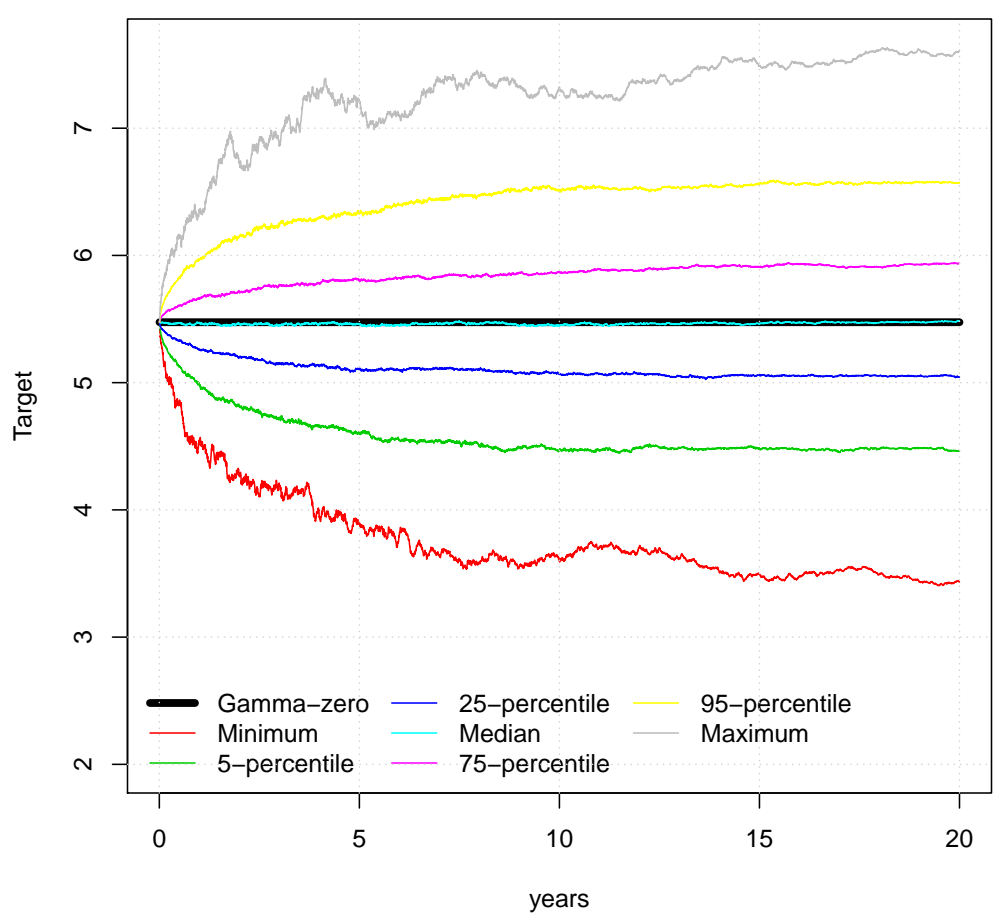

Figure 4: Statistics of time-varying targets for the naive approach, $\gamma_{t, x}$, and constant target for precommitment approach, $\gamma_{t_{0}, x_{0}}$

From Figures 1-4 we observe what follows.

- On average the wealth growth over time is exactly the same under the two approaches, that is consistent with Proposition 2 (Figure 1).

- The standard deviation of wealth is lower with precommitment than with the naive approach (Figure 1).

- In the worst cases, the precommitment wealth behaves much worse than the naive wealth (Figure 1). On the other hand, in the most favorable cases (at least top 25\%), the naive wealth behaves better than the precommitment wealth (Figure 1). 
- The two paths of the risky asset that lead to the worst PC-wealth and the worst Nwealth look quite similar one another, and the same applies for the two paths of the risky asset that lead to the best PC-wealth and the best $\mathrm{N}$-wealth (Figure 2, top-left graph).

- The time-varying target for the naive approach adjusts to market returns and increases over time in the two best cases (best PC-wealth and best $\mathrm{N}$-wealth), while it decreases over time in the two worst cases (worst PC-wealth and worst N-wealth) (Figure 2, top-right graph).

- In both the worst case for the precommitment wealth and the worst case for the naive wealth, the precommitment wealth behaves worse than the naive wealth, and the gap is larger in the worst case for the PC-wealth, as expected (Figure 2, bottom graphs). In both the best case for the precommitment wealth and the best case for the naive wealth, the naive wealth behaves better than the precommitment wealth, and the gap is larger in the best case for the naive wealth, as expected (Figure 2, bottom graphs).

- On average, the optimal portfolio of the precommitment strategy contains less risky asset than the naive strategy (Figure 3).

- The optimal share invested in the risky asset according to the precommitment strategy is highly more volatile than in the naive case (Figure 3).

- The optimal share invested in the risky asset on average is decreasing over time (Figure 3). ${ }^{6}$

- As expected from Proposition 3, the time-varying targets are on average equal to the constant precommitment target. Furthermore, the time-varying targets are symmetrically distributed around the mean (Figure 4).

The fact that the variance of wealth is lower with the precommitment strategy is consistent with the theory: the precommitment strategy minimizes the variance of the final wealth

\footnotetext{
${ }^{6}$ This decreasing trend occurs also in the case $r<\xi^{2}$, when instead the amount is increasing (see Equation $(24))$.
} 
given the same expected final wealth; therefore, any other strategy/portfolio that produces the same expected final wealth should produce a larger variance of final wealth.

Quite interestingly, however, we observe that in the worst cases for both the naive and the precommitment wealth, the precommitment strategy produces a lower wealth than the naive strategy. This outcome is both interesting and somehow unexpected, given that the precommitment strategy provides the lowest variance of final wealth. This only seeming contradiction is due to the fact that the naive strategy adjusts the final target at each time according to renovated circumstances. Instead, the precommitment strategy keeps the final target fixed over time. Indeed, when the market performance is bad, the fund is low; if the target remains fixed and too high compared to current wealth, the investment in the risky asset becomes important and this pushes the fund further down if the unfavorable market returns persist. If, instead, the target is regularly adjusted to current wealth and decreases when the fund falls down, the investment in stocks does not need to be so remarkable, and the potential loss from persisting bad market performance is reduced. Thus, the dynamically optimal strategy, which accounts for a time-varying target, seems to allow a more effective reaction against unfavorable market conditions that last for a long period, while the precommitment strategy does not. ${ }^{7}$

In addition, in the scenarios that lead to the best cases for both the naive and the precommitment wealth, the naive strategy produces a larger wealth than the precommitment strategy. This outcome is quite interesting too, and is again explained by the feature of the naive strategy to adjust the target. Indeed, when returns are high for a long period, the naive strategy implies a target which is higher and higher. Accordingly, this strategy leads to a larger final wealth than the precommitment strategy which, instead, is characterized by a constant target. Finally, we can conclude that the feature of the naive strategy to adjust the targets according to changing market conditions gives an extra reward to the pensioner in the presence of extreme scenarios of market returns, both in the good and in the bad

\footnotetext{
${ }^{7}$ On the other hand, whether the naive strategy always yields a more effective reaction in a period of bad market returns can be debatable. This is true when the bad returns keep on also in the future, because the precommitment strategy keeps on investing in the risky asset when returns are poor because of the unchanged high target (in that sense the precommitment approach is said to be contrarian, see Forsyth and Vetzal; 2017). But if a (short) period of bad returns is then followed by a period of good returns, the precommitment strategy might turn out to be better off than the naive one.
} 
direction.

The heavier investment in stocks of the precommitment strategy in bad scenarios is confirmed by the higher volatility of the precommitment investment strategy with respect to the naive strategy, that instead turns out to be more stable.

It must be noted that a similar result was observed by Forsyth and Vetzal (2017), in a comparison between precommitment strategy and constant proportions strategy: they, too, found that smallest values for the precommitment strategy were notably lower than the smallest values for the constant proportion strategy. They also found that the difference between the two strategies in the bad market scenarios was wider in the presence of leverage (unconstrained strategy) than with a no-leverage constraint. Therefore, it is likely that the remarkable difference we observe in the worst market scenarios between the precommitment and the naive strategy would be reduced should some no-short-selling constraints be imposed in the model.

Finally, we have run other simulations with a more and a less ambitious target (i.e. a target equal to $\kappa x_{0}^{T}$ with $\kappa>1.2$ and $\left.\kappa<1.2\right)$. When the target is changed, the quality of the result does not change, while the magnitude is modified according to the obvious financial intuition: higher (lower) target is pursued by investing higher (lower) percentage of wealth in the risky asset.

\section{Concluding remarks}

In this work we have solved the mean-variance portfolio allocation problem for a defined contribution pension scheme in an arbitrage free and complete market driven by any number of stochastic state variables and having any number of risky assets. We have provided and investigated the solution to the asset allocation problem with two common methods, the precommitment and the dynamically optimal naive approaches. While the precommitment approach has been deeply studied and applied in the literature, to the best of our knowledge this is the first paper that (i) provides the dynamically optimal naive strategy in a general model with many state variables and many assets; and (ii) thoroughly investigates the dynamically optimal naive approach in the framework of a DC pension fund.

The precommitment method is equivalent to fixing a given target at the initial time 
and keeping it unchanged over time. The dynamically optimal method is based on this precommitment strategy, where we modify the target at each instant in time, as if we were solving a newly starting optimization problem.

From the theoretical point of view, we prove that in the Black and Scholes market the expected wealth under the two strategies is the same. Moreover, we prove that the expected value of the time-varying target of the dynamically optimal strategy coincides with the constant target of the precommitment strategy.

Merits and weaknesses of the two strategies are further investigated via numerical simulations, which show that: (i) the precommitment portfolio contains less risky asset than the naive strategy; (ii) the amount of money invested in the precommitment risky portfolio is highly more volatile than in the naive case; (iii) as expected, the variance of wealth is lower with the precommitment strategy than with the naive one; (iv) interestingly, under scenarios of extreme market returns (either good or bad) that drive an extreme (rich or poor) performance in terms of the final wealth, the naive strategy allows a more effective reaction because of the continuous adjustment of the final target: in particular, in a bear market phase the naive strategy provides a better hedge against losses, while in a bull market phase it allows to exploit the high returns. These results might be of help to actuaries and investment managers of DC pension funds.

This paper leaves room for further research. In particular, the numerical analysis of a market with stochastic interest rate and stochastic contributions would be worth investigation. More importantly, introducing a variable risk aversion $\alpha_{t}$, and studying its impact on the precommitment and the naive strategies would also be quite interesting. This would also allow comparison with Bensoussan et al. (2019) and Björk et al. (2014), who find the Nash equilibrium strategy in the presence of a variable risk aversion. 


\section{A Proof of Proposition 1}

Problem (14) can be recast as a static problem where the choice variable is the final wealth:

$$
\begin{aligned}
& \inf _{X(T)} \mathbb{E}_{t_{0}}\left[\frac{1}{2}(X(T)-\gamma)^{2}\right] \\
& \text { s.t. } \quad \mathbb{E}_{t_{0}}^{\mathbb{Q}}\left[-\int_{t_{0}}^{T} c(s, z) e^{-\int_{t_{0}}^{s} r(u, z) d u} d s+X(T) e^{-\int_{t_{0}}^{T} r(u, z) d u}\right] \leq x_{0} .
\end{aligned}
$$

The Lagrangian function of this problem is

$$
\begin{aligned}
\mathcal{L} & =\mathbb{E}_{t_{0}}\left[\frac{1}{2}\left(X(T)-\gamma_{t_{0}, x_{0}}\right)^{2}+\lambda X(T) e^{-\int_{t_{0}}^{T} r(u, z) d u} m\left(t_{0}, T\right)\right] \\
& -\lambda\left(x_{0}+\int_{t_{0}}^{T} \mathbb{E}_{t_{0}}^{\mathbb{F} s}[c(s, z)] B\left(t_{0}, s\right) d s\right)
\end{aligned}
$$

where $\lambda$ is the Lagrangian multiplier. The derivative of $\mathcal{L}$ with respect to $X(T)$ must be set to zero for each state of the world, i.e.

$$
X^{*}(T)=\gamma_{t_{0}, x_{0}}-\lambda e^{-\int_{t_{0}}^{T} r(u, z) d u} m\left(t_{0}, T\right)
$$

Now, $\lambda$ is computed from the constraint in (27) where $X^{*}(T)$ is substituted, and the inequality is replaced by the equality (since we want the solution to be compatible with the minimum amount of initial wealth):

$$
\lambda=\frac{\gamma_{t_{0}, x_{0}} B\left(t_{0}, T\right)-\int_{t_{0}}^{T} \mathbb{E}_{t_{0}}^{\mathbb{F}_{s}}[c(s, z)] B\left(t_{0}, s\right) d s-x_{0}}{\mathbb{E}_{t_{0}}\left[e^{-2 \int_{t_{0}}^{T} r(u) d u} m^{2}\left(t_{0}, T\right)\right]}
$$

By defining the stochastic process $\Phi(t, T)$ as in (16), we can write

$$
\lambda=\frac{\gamma_{t_{0}, x_{0}} B\left(t_{0}, T\right)-\int_{t_{0}}^{T} \mathbb{E}_{t_{0}}^{\mathbb{F}_{s}}[c(s, z)] B\left(t_{0}, s\right) d s-x_{0}}{\mathbb{E}_{t_{0}}\left[e^{-2 \Phi\left(t_{0}, T\right)}\right]}
$$


or

$$
\lambda=\frac{B\left(t_{0}, T\right)}{\mathbb{E}_{t_{0}}\left[e^{-2 \Phi\left(t_{0}, T\right)}\right]}\left(\gamma_{t_{0}, x_{0}}-\frac{x_{0}+\int_{t_{0}}^{T} \mathbb{E}_{t_{0}}^{\mathbb{F}_{s}}[c(s, z)] B\left(t_{0}, s\right) d s}{B\left(t_{0}, T\right)}\right)
$$

In the optimal solution, the constraint (27) must hold at any instant in time:

$$
X^{*}(t)=-\int_{t}^{T} \mathbb{E}_{t}^{\mathbb{F}_{s}}[c(s, z)] B(t, s) d s+\mathbb{E}_{t}\left[X^{*}(T) e^{-\int_{t}^{T} r(u, z) d u} m(t, T)\right]
$$

If the optimal final wealth (28) is plugged into this equation we have:

$$
X^{*}(t)=-\int_{t}^{T} \mathbb{E}_{t}^{\mathbb{F} s}[c(s, z)] B(t, s) d s+\gamma_{t_{0}, x_{0}} B(t, T)-\lambda m\left(t_{0}, t\right) e^{-\int_{t_{0}}^{t} r(u, z) d u} \mathbb{E}_{t}\left[e^{2 \Phi(t, T)}\right]
$$

Now, the passages are as follows:

1. $d X^{*}(t)$ is found through Itō's lemma on (29) (differentiating w.r.t. $m\left(t_{0}, t\right)$ and $z(t)$ );

2. $\lambda m\left(t_{0}, t\right) e^{-\int_{t_{0}}^{t} r(u, z) d u} \mathbb{E}_{t}\left[e^{2 \Phi(t, T)}\right]$ is substituted into the diffusion term of $d X^{*}(t)$ from $(29)$;

3. this diffusion term is set equal to the diffusion term of investor's equation in (7) in order to find the portfolio which replicates the optimal wealth. Such a portfolio is given by $(20)$.

\section{B Proof of Proposition 2}

If we plug the precommitment strategy (22) in the wealth (8)we get the following dynamics for the precommitment wealth $\hat{X}(t)$

$d \hat{X}(t)=\left\{\hat{X}(t) r+c+\xi^{2}\left[x_{0} e^{r\left(t-t_{0}\right)}+\frac{c}{r}\left(e^{r\left(t-t_{0}\right)}-1\right)-\hat{X}(t)+\frac{1}{2 \alpha} e^{\xi^{2}\left(T-t_{0}\right)-r(T-t)}\right]\right\} d t+(\ldots) d W(t)$.

By taking the expectation at time $t_{0}$ given the wealth $x_{0}$, we get the following linear ordinary differential equation (ODE) for $\mathbb{E}_{t_{0}}[\hat{X}(t)]$ : 


$$
\frac{d \mathbb{E}_{t_{0}}[\hat{X}(t)]}{d t}=\left(r-\xi^{2}\right) \mathbb{E}_{t_{0}}[\hat{X}(t)]+b(t),
$$

where we have swapped the expected value and the derivative operators, and in which

$$
b(t)=c+\xi^{2} x_{0} e^{r\left(t-t_{0}\right)}+\xi^{2} \frac{c}{r}\left(e^{r\left(t-t_{0}\right)}-1\right)+\frac{\xi^{2}}{2 \alpha} e^{\xi^{2}\left(T-t_{0}\right)-r(T-t)}
$$

with initial condition

$$
\hat{X}\left(t_{0}\right)=x_{0} .
$$

The solution of the linear ODE (31) with the initial condition (32) is the expectation of the precommitment wealth:

$$
\mathbb{E}_{t_{0}}[\hat{X}(t)]=x_{0} e^{r\left(t-t_{0}\right)}+\frac{c}{r}\left(e^{r\left(t-t_{0}\right)}-1\right)+\frac{1}{2 \alpha} e^{-r(T-t)}\left(e^{\xi^{2}\left(T-t_{0}\right)}-e^{\xi^{2}(T-t)}\right)
$$

By following the same procedure for the dynamically optimal wealth (plugging (24) into (8), and taking expectation), we get the following ODE for the expected dynamically optimal wealth

$$
\frac{d \mathbb{E}_{t_{0}}[\tilde{X}(t)]}{d t}=r \mathbb{E}_{t_{0}}[\tilde{X}(t)]+c+\xi^{2} \frac{1}{2 \alpha} e^{\left(\xi^{2}-r\right)(T-t)},
$$

with the same initial condition (32). By solving (34) with initial condition (32), we get the following expectation of the dynamically optimal naive wealth:

$$
\mathbb{E}_{t_{0}}[\tilde{X}(t)]=x_{0} e^{r\left(t-t_{0}\right)}+\frac{c}{r}\left(e^{r\left(t-t_{0}\right)}-1\right)+\frac{1}{2 \alpha} e^{-r(T-t)}\left(e^{\xi^{2}\left(T-t_{0}\right)}-e^{\xi^{2}(T-t)}\right)
$$

and comparing (33) with (35), we get the claim (25). 


\section{Proof of Proposition 3}

The analogue of $\gamma_{t_{0}, x_{0}}$ (19) at time $t$ with wealth $x$ is

$$
\gamma_{t, x}=\frac{1}{2 \alpha} e^{\xi^{2}(T-t)}+x e^{r(T-t)}+\frac{c}{r}\left(e^{r(T-t)}-1\right)
$$

Therefore

$$
\mathbb{E}_{t_{0}}\left[\gamma_{t, \tilde{X}(t)}\right]=\frac{1}{2 \alpha} e^{\xi^{2}(T-t)}+\frac{c}{r}\left(e^{r(T-t)}-1\right)+\mathbb{E}_{t_{0}}[\tilde{X}(t)] e^{r(T-t)}
$$

By plugging (35) into (36), and recalling (19), we get

$$
\mathbb{E}_{t_{0}}\left[\gamma_{t, \tilde{X}(t)}\right]=x_{0} e^{r\left(T-t_{0}\right)}+\frac{c}{r}\left(e^{r\left(T-t_{0}\right)}-1\right)+\frac{1}{2 \alpha} e^{\xi^{2}\left(T-t_{0}\right)}=\gamma_{t_{0}, x_{0}},
$$

that is claim (26). 


\section{References}

Basak, S. and Chabakauri, G. (2010). Dynamic mean-variance asset allocation, Review of Financial Studies 23: 2970-3016.

Bensoussan, A., Wong, K. and Yam, S. (2019). A paradox in time-consistency in the meanvariance problem?, Finance and Stochastics 23: 173-207.

Björk, T. (1998). Arbitrage Theory in Continuous Time, Oxford University Press.

Björk, T., Khapko, M. and Murgoci, A. (2017). On time-inconsistent stochastic control in continuous time, Finance and Stochastics 21: 331-360.

Björk, T., Murgoci, A. and Zhou, X. (2014). Mean variance portfolio optimization with state dependent risk aversion, Mathematical Finance 24: 1-24.

Cong, F. and Oosterlee, C. (2016). On pre-commitment aspects of a time-consistent strategy for a mean-variance investor, Journal of Economic Dynamics and Control 70: 178-193.

Cui, X., Li, D. and Shi, Y. (2017). Self-coordination in time inconsistent stochastic decision problems: A planner-doer game framework, Journal of Economic Dynamics and Control 75: $91-113$.

Cui, X., Li, D., Wang, S. and Zhu, S. (2012). Better than dynamic mean-variance: time inconsistency and free cash flow stream, Mathematical Finance 22: 346-378.

Forsyth, P. A. and Vetzal, K. R. (2017). Robust asset allocation for long-term targetbased investing, International Journal of Theoretical and Applied Finance 20: 1750017 1175001732.

Guan, G. and Liang, Z. (2015). Mean-variance efficiency of DC pension plan under stochastic interest rate and mean-reverting returns, Insurance: Mathematics and Economics 61: 99109.

He, L. and Liang, Z. (2013). Optimal investment strategy for the DC plan with the return of premiums clauses in a mean-variance framework, Insurance: Mathematics and Economics 53: 643-649. 
Karatzas, I. and Shreve, S. (1998). Methods of Mathematical Finance, Springer.

Menoncin, F. and Vigna, E. (2017). Mean-variance target-based optimisation for defined contribution pension schemes in a stochastic framework, Insurance: Mathematics and Economics 76: 172-184.

Pedersen, J. and Peskir, G. (2017). Optimal mean-variance portfolio selection, Mathematics and Financial Economics 11: 137-160.

Pollak, R. A. (1968). Consistent planning, The Review of Economic Studies 35: 201-208.

Shi, Y., Li, X. and Cui, X. (2017). Better than pre-committed optimal mean-variance policy in a jump diffusion market, Mathematical Methods of Operations Research 85: 327-347.

Strotz, R. H. (1956). Myopia and inconsistency in dynamic utility maximization, The Review of Economic Studies 23: 165-180.

Vigna, E. (2014). On efficiency of mean variance based portfolio selection in defined contribution pension schemes, Quantitative Finance 14: 237-258.

Wang, J. and Forsyth, P. A. (2011). Continuous time mean variance asset allocation: A time-consistent strategy, European Journal of Operational Research 209: 184-201.

Wu, H., Zhang, L. and Chen, H. (2015). Nash equilibrium strategies for a defined contribution pension management, Insurance: Mathematics and Economics 62: 202-214.

Yao, H., Lai, Y., Ma, Q. and Jian, M. (2014). Asset allocation for a DC pension fund with stochastic income and mortality risk: a multi-period mean-variance framework, Insurance: Mathematics and Economics 54: 84-92.

Yao, H., Yang, Z. and Chen, P. (2013). Markowitz's mean-variance defined contribution pension fund management under inflation: a continuous-time model, Insurance: Mathematics and Economics 53: 851-863.

Zhou, X. and Li, D. (2000). Continuous-time mean-variance portfolio selection: A stochastic LQ framework, Applied Mathematics and Optimization 42: 19-33. 